\title{
Cefiderocol: A Review in Serious Gram-Negative Bacterial Infections
}

\author{
Yahiya Y. Syed ${ }^{1}$
}

Accepted: 26 July 2021 / Published online: 24 August 2021

(c) Springer Nature 2021, corrected publication 2021

\begin{abstract}
Intravenous cefiderocol $\left(\right.$ Fetroja $^{\circledR} ;$ Fetcroja $\left.^{\circledR}\right)$ is the first siderophore cephalosporin approved for the treatment of adults with serious Gram-negative bacterial infections. Cefiderocol is stable against all four Ambler classes of $\beta$-lactamases (including metallo- $\beta$-lactamases) and exhibits excellent in vitro activity against many clinically relevant Gram-negative pathogens, including multidrug resistant strains. In randomized, double-blind clinical trials, cefiderocol was noninferior to imipenem/ cilastatin for the treatment of complicated urinary tract infections (cUTI) and to meropenem for nosocomial pneumonia. Furthermore, in a pathogen-focused clinical trial in patients with carbapenem-resistant (CR) infections, cefiderocol showed comparable efficacy to best available therapy (BAT), albeit all-cause mortality rate was higher in the cefiderocol arm, the cause of which has not been established. Cefiderocol had a good tolerability and safety profile in clinical trials. Thus cefiderocol is a novel, emerging, useful addition to the current treatment options for adults with susceptible Gram-negative bacterial infections (including cUTI and nosocomial pneumonia) for whom there are limited treatment options.
\end{abstract}

\section{Plain Language Summary}

Infections caused by carbapenem-resistant (CR) Enterobacterales and nonfermenters (such as Pseudomonas, Acinetobacter, Stenotrophomonas, Burkholderia) are a major global health threat. Cefiderocol, a cephalosporin with activity against CR Enterobacterales and nonfermenters, uses the bacteria's own iron uptake system to gain cell entry, like a Trojan horse. Once inside, the drug disrupts the formation of the bacterial cell wall, killing the bacteria. Cefiderocol is approved for the treatment of serious Gram-negative bacterial infections. In clinical trials, cefiderocol was effective versus carbapenems or best available therapy for complicated urinary tract infections, nosocomial pneumonia and bloodstream infections/sepsis, including those caused by CR bacteria. The drug had a good tolerability and safety profile. Thus, cefiderocol is a useful addition to the current treatment options for adults with cefiderocol-susceptible Gram-negative bacterial infections for whom there are limited treatment options.

Digital Features for this Adis Drug Evaluation can be found at https://doi.org/10.6084/m9.figshare.14920275.
The manuscript was reviewed by: $\boldsymbol{H}$. S. Sader, Antimicrobial Resistance Surveillance, JMI Laboratories, North Liberty, IA, USA; E. Wenzler, Department of Pharmacy Practice, University of Illinois at Chicago, Chicago, IL, USA; R. Zaragoza, Intensive Care Unit (Sepsis), Hospital Universitario Doctor Peset, Valencia, Spain.

Yahiya Y. Syed

demail@springer.com

1 Springer Nature, Private Bag 65901, Mairangi Bay, Auckland 0754, New Zealand

\section{Introduction}

Multidrug-resistant (MDR) Gram-negative pathogens are causing an increasing number of infections, including complicated urinary tract infections (cUTI) [1], nosocomial pneumonia [2] and bloodstream infections (BSI)/ sepsis [3]. The WHO has designated carbapenem-resistant (CR) strains of Enterobacterales, Pseudomonas aeruginosa and Acinetobacter baumannii, and third-generation cephalosporin-resistant Enterobacterales, as 'priority 1: critical' pathogens for which new treatments are urgently needed [4]. The US CDC have identified CR Enterobacterales and A. baumannii as urgent threats and MDR P. aeruginosa as a serious threat [5]. Stenotrophomonas maltophilia is also an emerging MDR opportunistic pathogen 


\section{Cefiderocol: clinical considerations}

First siderophore cephalosporin.

Excellent in vitro activity; stable vs all Ambler classes of $\beta$-lactamases (including metallo- $\beta$-lactamases).

Noninferior efficacy to carbapenems for cUTI and nosocomial infections; comparable efficacy to BAT in serious CR infections.

Good tolerability and safety profile.

that confers intrinsic resistance to multiple antibacterial classes, including $\beta$-lactams [6].

Carbapenem resistance mechanisms include $\beta$-lactamases, efflux pumps, and porin loss or mutation [7]. Novel $\beta$-lactam/ $\beta$-lactamase-inhibitor combinations (ceftazidime-avibactam, ceftolozane-tazobactam, meropenem-vaborbactam, imipenem/cilastatin-relebactam) and non- $\beta$-lactam antibacterials (e.g. plazomicin) overcome some of these resistance mechanisms [8]. However, none of the new $\beta$-lactam $/ \beta$-lactamase inhibitor combinations are stable against metallo- $\beta$-lactamases and ceftolozane-tazobactam is not stable against KPC $[8,9]$. Thus, additional antibacterials against $\mathrm{CR}$ pathogens remains an unmet medical need [10].

Cefiderocol (Fetroja ${ }^{\circledR}$; Fetcroja ${ }^{\circledR}$ ), an intravenously infused siderophore cephalosporin with a unique mechanism of bacterial cell entry, is approved in the EU and the USA for the treatment of adults with Gram-negative bacterial infections (Sect. 6). This article reviews the efficacy and tolerability of cefiderocol in the approved indications and overviews its pharmacological properties.

\section{Pharmacodynamic Properties}

Cefiderocol is actively transported into the periplasmic space of Gram-negative bacteria through the bacterial siderophore iron uptake system, as well as through passive diffusion via outer membrane porin channels [11-13]. Iron transporters PiuA in P. aeruginosa, and CirA and Fiu in Escherichia coli have been shown to contribute to the transport of cefiderocol across the outer membrane [14]. Once inside, cefiderocol binds to penicillin binding proteins (PBPs), primarily PBP3, and inhibits peptidoglycan cell wall synthesis, causing cell death [11-13]. Cefiderocol is structurally related to ceftazidime and cefepime. While all three compounds have an aminothiazole ring at $\mathrm{C} 7$, which confers stability against $\beta$-lactamases, cefiderocol has a catechol side chain at $\mathrm{C} 3$ that acts as an iron-binding siderophore. Thus, it mimics bacterial siderophore and gains cell entry, like a 'Trojan horse' [11-13]. The unique structure of cefiderocol, along with low catalytic efficiencies of carbapenemases against this drug, confers stability against all four Ambler classes of $\beta$-lactamases $[15,16]$. Truncation or loss of porin channels and upregulation of efflux pumps had no marked effect on the activity of cefiderocol $[14,17,18]$. Cefiderocol has no clinically relevant activity against Gram-positive or anaerobic bacteria [10].

\subsection{Antibacterial Activity}

Due to its iron transporter-based mechanism of cell entry, in vitro susceptibility testing of cefiderocol is performed using iron-depleted cation-adjusted Mueller-Hinton broth, recommended by the CLSI and the EUCAST. CLSIapproved cefiderocol minimum inhibitory concentration (MIC) breakpoints are $\leq 4,8$ and $\geq 16 \mathrm{mg} / \mathrm{L}$ (susceptible, intermediate and resistant, respectively) for Enterobacterales, $P$. aeruginosa and A. baumannii, and $\leq 1$ and $>1$ $\mathrm{mg} / \mathrm{L}$ (susceptible and nonsusceptible) for S. maltophilia (based on limited clinical data) [19]. Cefiderocol breakpoints approved by CLSI, US FDA and EUCAST differ markedly, which could impact susceptibility or resistance reporting [20, 21], particularly for NDM-producing carbapenem-nonsusceptible Enterobacterales and IMP-producing P. aeruginosa [21]. Disk diffusion method could also be a convenient alternative to the broth microdilution method for cefiderocol susceptibility testing [20]. Cefiderocol susceptibility rates reported in this article are based on the CLSI breakpoints, using the broth microdilution method.

The in vitro activity of cefiderocol and relevant comparators against Gram-negative clinical isolates was evaluated in two multinational surveillance programmes, SIDERO-WT [18, 22-26] and SIDERO-CR [27], as part of its preclinical development. In SIDERO-WT, > 30,000 isolates were collected from North American and European hospitals in three consecutive studies during 2014-2015, 2015-2016 and 2016-2017. In SIDERO-CR, 1873 carbapenem-nonsusceptible isolates, including Enterobacterales, MDR A. baumannii, MDR P. aeruginosa, and S. maltophilia were collected globally between 2014 and 2016. In vitro data are also available from other studies assessing cefiderocol against a wide range of clinically important isolates collected globally [14, 28-30] and from Canada [31], Germany [32], Greece [33], Japan [34, 35], Spain [36], Taiwan [37], the UK [38] and the USA [17, 39-42].

In a pooled analysis of SIDERO-WT studies, MIC required to inhibit $90 \%$ of isolates $\left(\mathrm{MIC}_{90}\right)$ ranged from 0.12 to $2 \mathrm{mg} / \mathrm{L}$ for Enterobacterales (E. coli, Klebsiella pneumonia, Klebsiella oxytoca, Citrobacter freundii, Citrobacter koseri, Enterobacter cloacae, Enterobacter aerogenes, Serratia marcescens), Nonfermenters (P. aeruginosa, A. 
baumannii, S. maltophilia, Burkholderia cepacia complex) and Proteaceae (Proteus mirabilis, Proteus vulgaris, Providencia rettgeri Morganella morganii); $95-100 \%$ of these isolates were susceptible to cefiderocol. In the cefiderocol MIC distribution range, peak MIC values lie between 0.06 and $0.12 \mathrm{mg} / \mathrm{mL}$ for most Enterobacterales, $P$. aeruginosa, A. baumannii and S. maltophilia isolates [43]. Cefiderocol $\mathrm{MIC}_{90}$ values remained stable over the 3-year surveillance period for all species, except for A. baumannii for which values increased over time but were within the susceptible range [26]. Cefiderocol was the most potent agent among the six comparators (cefepime, ceftazidime-avibactam, ceftolozane-tazobactam, ciprofloxacin, colistin and meropenem) tested [18, 22-26]. Against S. maltophilia, $\mathrm{MIC}_{90}$ values were $0.25 \mathrm{mg} / \mathrm{L}$ for cefiderocol compared with $\geq 8 \mathrm{mg} / \mathrm{L}$ for colistin and ciprofloxacin and $\geq 64 \mathrm{mg} / \mathrm{L}$ for other comparators. Cefiderocol $\mathrm{MIC}_{90}$ values against B. cepacia complex were $\geq 16$-fold lower than those for comparators [18, 22-26]. SIDERO-WT findings are supported by other studies $[17,31,32,37,40,41]$.

In a SIDERO-WT subpopulation analysis, cefiderocol showed potent activity against meropenem-nonsusceptible strains of Enterobacterales, $P$. aeruginosa, Acinetobacter spp. and B. cepacia (Table 1). It was also active against isolates nonsusceptible to one or more comparator drugs and against difficult-to-treat resistant strains (Table 1) [23-25]. Against Enterobacterales and $P$. aeruginosa strains nonsusceptible to both ceftazidime-avibactam and ceftolozane-tazobactam, cefiderocol susceptibility was $98.2 \%$ and $100 \%$ [23].

These findings were further supported by the SIDEROCR study in which cefiderocol demonstrated potent activity against meropenem-nonsusceptible Enterobacterales, MDR $P$. aeruginosa and MDR A. baumannii (Table 1) [27]. Furthermore, cefiderocol was active against meropenem-nonsusceptible Enterobacterales and MDR $P$. aeruginosa isolates nonsusceptible to ceftazidime-avibactam and ceftolozane-tazobactam (Table 1) [27].

Overall, cefiderocol was more potent than cefepime, ceftazidime-avibactam, ceftolozane-tazobactam and ciprofloxacin against isolates nonsusceptible to meropenem and/or other drug(s) (Table 1) [23, 27]. Colistin was the only other tested agent with consistent activity against these isolates, and cefiderocol was active against colistin-nonsusceptible isolates [23, 27]. In SIDERO-CR, 13.0\% of 1613 isolates were nonsusceptible to colistin and $96.7 \%$ of these isolates were susceptible to cefiderocol [27]. In other studies, cefiderocol susceptibility rates ranged from 86 to $100 \%$ against CR strains of Enterobacterales, $P$. aeruginosa, A. baumannii and S. maltophilia [17, 28, 31, 33, 37, 38, 40, 41]. In MDR strains of Enterobacterales, $P$. aeruginosa and S. maltophilia, cefiderocol susceptibility was $>95 \%[33,36,39,40]$.
Cefiderocol had potent activity against Gram-negative bacteria producing all four Ambler classes of $\beta$-lactamases, including extended spectrum $\beta$-lactamases (ESBL) and carbapenemases $[14,17,18,29,32,35,36,38,41,42$, 44-48]. In meropenem-resistant or -intermediate isolates in SIDERO-WT $(n=1272)$ [18] and SIDERO-CR $(n=1651)$ [45], 97-98\% of $\beta$-lactamase-producing Enterobacterales, $P$. aeruginosa and $A$. baumannii isolates were susceptible to cefiderocol. Cefiderocol $\mathrm{MIC}_{90}$ values were $\leq 4 \mathrm{mg} / \mathrm{L}$ for isolates positive for KPC, GES, IMP, VIM, OXA-23 and OXA-58, and $8 \mathrm{mg} / \mathrm{L}$ for those positive for NDM or OXA-24/40 [18, 45]. In vitro activity of cefiderocol and comparator agents against individual $\beta$-lactamase genotypes is summarized in Table 2. Cefiderocol was the only agent active against all genotypes, while ceftazidime-avibactam and colistin were active against KPC and OXA genotypes (Table 2).

In other studies, cefiderocol susceptibility rates were 94-100\% for ESBL-producing Enterobacterales [31, $32,40], 100 \%$ for S. maltophilia producing L1 and L2 $\beta$-lactamases [41], $\approx 72 \%$ for NDM-producing Enterobacterales and P. aeruginosa [38] and 73\% for PER-producing $P$. aeruginosa [38]. Cefiderocol was stable against chromosomal AmpC $\beta$-lactamases of P. aeruginosa and E. cloacae, and had a low propensity to induce AmpC $\beta$-lactamases in these isolates [47]. In Enterobacterales harbouring ESBL or AmpC $\beta$-lactamases and porin loss, cefiderocol susceptibility was $88-100 \%$ [38].

\subsubsection{In Vivo Activity}

The in vitro activity of cefiderocol correlated well with its bactericidal effects in murine models of thigh or lung infection [49-56], respiratory tract infection [57] and ventilatorassociated pneumonia (VAP) [58], caused by Enterobacterales, $P$. aeruginosa and A. baumannii or S. maltophilia, including CR strains [56-58]. Data from representative animal studies are discussed in Sect. 2.2.

\subsubsection{Resistance Mechanisms}

Cefiderocol may have a low potential for resistance development. In SIDERO-WT [59] and SIDERO-CR [60], 0.56\% and $3.8 \%$ of isolates $(n=28,629$ and 1837$)$ were nonsusceptible to cefiderocol (i.e. $\mathrm{MIC} \geq 4 \mathrm{mg} / \mathrm{L}$ ); the most common nonsusceptible isolates were PER-producing A. baumannii and NDM-producing Enterobacterales. While PER or NDM could contribute to cefiderocol resistance, they alone may not be sufficient to cause the resistance [60-62]. Other possible mechanisms of resistance to cefiderocol include siderophore receptor gene truncation, PBP3 modification and AmpC $\beta$-lactamase mutations [59, 61, 63]. In E. coli strains, PBP3 modification by YRIN insertion was associated with 
Table 1 In vitro activity of cefiderocol against selected clinical isolates by susceptibility phenotype in SIDERO-WT and SIDERO-CR

Pathogen/susceptibility phenotype (no. of isolates)

$\mathrm{MIC}_{90}(\mathrm{mg} / \mathrm{L})\left[\%\right.$ susceptible $\left.^{\mathrm{a}}\right]$

\begin{tabular}{lllllll}
\hline FDC & FEP & CZA & $\mathrm{C} / \mathrm{T}$ & CIP & CST & MEM
\end{tabular}

\section{SIDERO-WT [23]}

Enterobacterales

\begin{tabular}{|c|c|c|c|c|c|c|c|}
\hline MEM-nonsusceptible (246) & 4 [99.6] & $>64[8.5]$ & $>64$ [78.5] & $>64$ [7.7] & $>8[12.6]$ & $>8[69.5]$ & $>64[0]$ \\
\hline CZA-nonsusceptible (57) & $4[98.2]$ & $>64[3.5]$ & $>64[0]$ & $>64[3.5]$ & $>8[17.5]$ & $8[84.7]$ & $>64[7.0]$ \\
\hline C/T-nonsusceptible (597) & $2[98.8]$ & $>64[25.6]$ & $8[90.8]$ & $>64[0]$ & $>8[38.4]$ & $>8[83.1]$ & $64[62.0]$ \\
\hline FEP-nonsusceptible (1002) & $2[99.5]$ & $>64[0]$ & $2[94.5]$ & $>64[55.7]$ & $>8$ [21.9] & $8[88.0]$ & $16[75.0]$ \\
\hline CIP-nonsusceptible (1299) & $2[99.7]$ & $>64$ [39.7] & $2[96.4]$ & $>64[71.7]$ & $>8[0]$ & $4[89.8]$ & $8[83.5]$ \\
\hline CST-nonsusceptible (930) & $1[99.8]$ & $16[87.1]$ & $1[99.2]$ & $4[89.1]$ & $4[85.8]$ & $>8[0]$ & 0.5 [91.9] \\
\hline Difficult-to-treat resistant ${ }^{\mathrm{b}}$ (573) [24] & $4[98.3]$ & & $>64[78.2]$ & $>64[2.05]$ & & $>8[68.2]$ & \\
\hline \multicolumn{8}{|l|}{ Pseudomonas aeruginosa } \\
\hline MEM-nonsusceptible (1416) [25] & $1[99.9]$ & 64 & 64 & $>64$ & $>8$ & 2 & 64 \\
\hline CZA-nonsusceptible (113) & $1[100]$ & $>64[5.3]$ & $>64[0]$ & $>64[21.2]$ & $>8[12.4]$ & $1[99.1]$ & $>64[5.3]$ \\
\hline C/T-nonsusceptible (111) & $1[100]$ & $>64[6.3]$ & $>64[19.8]$ & $>64[0]$ & $>8[12.6]$ & $1[99.1]$ & $>64[7.2]$ \\
\hline FEP-nonsusceptible (300) & $1[99.7]$ & $64[0]$ & $64[64.3]$ & $>64[65.3]$ & $>8[33.0]$ & $1[99.0]$ & $>64[30.3]$ \\
\hline CIP-nonsusceptible (424) & $1[99.8]$ & $32[52.6]$ & $64[76.7]$ & $>64[77.1]$ & $>8[0]$ & $1[99.1]$ & $>64[41.0]$ \\
\hline Difficult-to-treat resistant ${ }^{\mathrm{b}}$ (470) [24] & $1[99.8]$ & & $>64[49.5]$ & $>64[48.8]$ & & $2[98.3]$ & \\
\hline \multicolumn{8}{|l|}{ Acinetobacter species } \\
\hline MEM-nonsusceptible (2274) [25] & $2[94.0]$ & $>64$ & $>64$ & $>64$ & $>8$ & $>8$ & $>64$ \\
\hline FEP-nonsusceptible (602) & $2[94.2]$ & $>64[0]$ & $>64^{\mathrm{c}}$ & $>64^{c}$ & $>8[3.2]$ & $>8[82.6]$ & $>64[11.1]$ \\
\hline CIP-nonsusceptible (633) & $2[94.5]$ & $>64$ [7.9] & $>64^{\mathrm{c}}$ & $>64^{\mathrm{c}}$ & $>8[0]$ & $>8[82.1]$ & $>64[11.4]$ \\
\hline CST-nonsusceptible (114) & $2[99.1]$ & $>64$ [7.9] & $>64^{\mathrm{c}}$ & $>64^{\mathrm{c}}$ & $>8[0.9]$ & $>8[0]$ & $>64[2.6]$ \\
\hline Difficult-to-treat resistant $^{\mathrm{b}}$ (1794) [24] & $2[94.5]$ & & $>64^{\mathrm{c}}$ & $>64^{c}$ & & $8[84]$ & \\
\hline MEM-nonsusceptible B. cepacia complex (80) [25] & $2\left[90.0^{\mathrm{d}}\right]$ & $>64$ & 16 & $>64$ & $>8$ & $>8$ & 16 \\
\hline \multicolumn{8}{|l|}{ IDERO-CR [27] } \\
\hline \multicolumn{8}{|l|}{ Enterobacterales } \\
\hline MEM-nonsusceptible (1022) & $4[97.0]$ & $>64[2.8]$ & $>64[77.0]$ & $>64[1.7]$ & $>8[11.5]$ & $>8[77.8]$ & $>64[0]$ \\
\hline MEM- and C/T-nonsusceptible (1005) & 4 [91.9] & $>64[1.8]$ & $>64[76.6]$ & $>64[0]$ & $>8[10.4]$ & $>8[78.2]$ & $>64[0]$ \\
\hline MEM- and CZA-nonsusceptible (235) & $4[96.7]$ & $>64[1.3]$ & $>64[0]$ & $>64[0]$ & $>8[15.7]$ & $>8[83.8]$ & $32[0]$ \\
\hline \multicolumn{8}{|l|}{ P. aeruginosa } \\
\hline IDR (262) & $1[99.2]$ & $>64[13.7]$ & $>64[36.3]$ & $>64[24.1]$ & $>8[1.2]$ & 1 [99.6] & $>64[3.8]$ \\
\hline MDR and C/T-nonsusceptible (199) & $2[99.0]$ & $>64[6.0]$ & $>64[16.6]$ & $>64[0]$ & $>8[0.5]$ & $1[100]$ & $>64[1.5]$ \\
\hline MDR and CZA-nonsusceptible (167) & $2[98.8]$ & $>64[1.8]$ & $>64[0]$ & $>64[0.6]$ & $>8[0.6]$ & $1[100]$ & $>64[0]$ \\
\hline MDR A. baumannii (368) & $8[89.7]$ & $>64[3.3]$ & $>64$ & $>64$ & $>8[0]$ & $1[94.6]$ & $32[1.9]$ \\
\hline
\end{tabular}

$C / T$ ceftolozane-tazobactam, $C I P$ ciprofloxacin, $C S T$ colistin, $C Z A$ ceftazidime-avibactam, $F D C$ cefiderocol, $F E P$ cefepime, $M D R$ multidrug resistant, $M E M$ meropenem, $M I C_{90}$ minimum inhibitory concentration required to inhibit $90 \%$ of isolates

${ }^{a}$ Reported only where available; ${ }^{b}$ Nonsusceptible to FEP, CIP and MEM according to CLSI breakpoints; ${ }^{c}$ Minimum inhibitory concentration breakpoints not established; ${ }^{\mathrm{d} B a s e d}$ on EUCAST breakpoint

a twofold increase in cefiderocol MIC (from 0.06 to 0.125 $\mathrm{ng} / \mathrm{L}$ ), compared with an eightfold increase for cefepime, ceftazidime, ceftolozane and ceftolozane/tazobactam, and a fourfold increase for ceftazidime/avibactam [64]. $p v d S$ gene mutation in $P$. aeruginosa was also associated with increased cefiderocol MICs, mainly due to reduced expression of iron transporters [65].

\subsection{Pharmacological Considerations}

The fraction of dosing interval during which the free drug concentration in plasma exceeds the MIC (\% $\mathrm{T}$ T $>$ MIC) is considered a reliable pharmacodynamic index for cefiderocol. In a murine thigh infection model with $P$. aeruginosa (cefiderocol MIC 0.063-0.5 mg/L), bacterial stasis and $1 \log _{10}$ and $2 \log _{10}$ reductions in colony forming unit (CFU) at $24 \mathrm{~h}$ were observed at cefiderocol mean $\% \mathrm{fT}>\mathrm{MIC}$ values of $76.3 \%, 81.9 \%$ and $88.2 \%$, respectively [54]. In the same model, a human-simulated cefiderocol regimen $(2 \mathrm{~g}$ 
Table 2 In vitro activity of cefiderocol against selected clinical isolates by $\beta$-lactamase genotype in SIDERO-WT and SIDERO-CR

\begin{tabular}{|c|c|c|c|c|c|c|c|}
\hline \multirow{2}{*}{$\begin{array}{l}\text { Pathogen/ } \beta \text {-lactamase } \\
\text { genotypes (no. of isolates) }\end{array}$} & \multicolumn{7}{|c|}{$\mathrm{MIC}_{90}(\mathrm{mg} / \mathrm{L})\left[\%\right.$ susceptible $\left.{ }^{\mathrm{a}}\right]$} \\
\hline & FDC & FEP & CZA & $\mathrm{C} / \mathrm{T}$ & CIP & CST & MEM \\
\hline \multicolumn{8}{|l|}{ Enterobacterales } \\
\hline KPC (644) [18, 45] & $2-4[98.1]$ & $>64[0]$ & $4[96]$ & $>64[0]$ & $>8[5.3]$ & $>8$ & $>64[0]$ \\
\hline $\operatorname{NDM}(162)[44,45]$ & $8[84-87.2]$ & $>64$ & $>64$ & $>64$ & $>8$ & 1 to $>8$ & $>64$ \\
\hline VIM (174) [44, 45] & $4[98.0]$ & $>64$ & $>64$ & $>64$ & $>8$ & 2 to $>8$ & $\geq 64$ \\
\hline OXA-48 $(168)[18,45]$ & $4[100]$ & $>64[12.5]$ & 4 to $>64[90.6]$ & $>64[3.1]$ & $>8[3.1]$ & $>8[78.1]$ & $\geq 64[0]$ \\
\hline VIM (174) $[44,45]$ & $4[98.0]$ & $>64$ & $>64$ & $>64$ & $>8$ & 2 to $>8$ & $\geq 64$ \\
\hline \multicolumn{8}{|l|}{ P. aeruginosa } \\
\hline VIM (256) $[44,45]$ & $0.5-1[100]$ & $>64$ & $>64$ & $>64$ & $>8$ to $>64$ & $1-2$ & $>8$ to $>64$ \\
\hline IMP (16) [44] & 1 & $>64$ & $>64$ & $>64$ & $>64$ & 2 & $>8$ \\
\hline \multicolumn{8}{|l|}{ A. baumannii } \\
\hline OXA-23 $(775)[18,45]$ & $1-2[92.2]$ & $>64.7[1.7]$ & $>64$ & $>64$ & $>8[0]$ & 1 to $>8[79.6]$ & $>64[0]$ \\
\hline OXA-24 (237) $[18,45]$ & $1-8[89.4]$ & $>64.7[11.3]$ & $>64$ & $>64$ & $>8[0]$ & $1[96.8]$ & $>64[0]$ \\
\hline OXA-58 (14) [18] & 1 & $>64[0]$ & $>64$ & $>64$ & $>8[0]$ & 1 [92.9] & $16[0]$ \\
\hline
\end{tabular}

$C / T$ ceftolozane-tazobactam, $C I P$ ciprofloxacin, $C S T$ colistin, $C Z A$ ceftazidime-avibactam, $F D C$ cefiderocol, $F E P$ cefepime, $M E M$ meropenem, $M I C_{90}$ minimum inhibitory concentration required to inhibit $90 \%$ of isolates

${ }^{\text {a }}$ Reported only where available

every $8 \mathrm{~h}, 3$-h infusion) produced bacterial stasis or $2 \log _{10}$ reduction in CFU at $24 \mathrm{~h}$ in $88 \%$ of $A$. baumannii $(n=16)$, $85 \%$ of $P$. aeruginosa $(n=20)$ and $77 \%$ of Enterobacterales $(n=31)$ isolates, with a cefiderocol MIC of $\leq 4 \mathrm{mg} / \mathrm{L}$ [55]. This regimen is predicted to have a $\% f \mathrm{~T}>\mathrm{MIC}$ of $96 \%$ and $80 \%$ for a MIC of 4 and $8 \mathrm{mg} / \mathrm{L}$ [55]. In a murine lung infection model, the mean $\% \mathrm{~T}$ $>$ MIC required for a $1 \log _{10}$ reduction in CFU was $64.4 \%$ for Enterobacterales, $70.3 \%$ for $P$. aeruginosa, $88.1 \%$ for A. baumannii and $53.9 \%$ for S. maltophilia [51]. With the humanized regimen, increasing the infusion period from 1 to $3 \mathrm{~h}$ increased $\% \mathrm{fT}>\mathrm{MIC}$ for MICs of $4 \mathrm{mg} / \mathrm{L}$ (from $75 \%$ to $100 \%$ ) and $8 \mathrm{mg} / \mathrm{L}$ (from $50 \%$ to $100 \%$ ) in immunocompetent rat models [57]. Results from an in vitro chemostat model were consistent with those from animal studies [34].

A population pharmacokinetic model showed that the recommended cefiderocol dosage regimen of $2 \mathrm{~g}$ every $8 \mathrm{~h}$ (3-h infusion), adjusted for kidney function, would provide adequate drug exposure in patients with serious infections (pneumonia, cUTI or BSI/sepsis) [66]. The probability of target attainment for $100 \% \mathrm{fT}>\mathrm{MIC}$ against MICs $\leq 4 \mathrm{mg} / \mathrm{L}$ was $>90 \%$ for different infections sites and kidney function groups, with the exception of BSI/sepsis and normal kidney function (86\%) [66].

\section{Pharmacokinetic Properties of Cefiderocol}

Cefiderocol showed linear pharmacokinetics within a 100-4000 mg dose range [67, 68]. Following multiple 3-h infusions of $2 \mathrm{~g}$ every $8 \mathrm{~h}$ (or kidney function-adjusted dosage), the mean maximum plasma concentration of cefiderocol was $115 \mathrm{mg} / \mathrm{L}$ in patients with cUTI and $111 \mathrm{mg} / \mathrm{L}$ in those with bacterial hospital-acquired pneumonia (HAP) or VAP; area under the plasma concentration-time curve (AUC) from time zero to $24\left(\mathrm{AUC}_{24}\right)$ was 1944 and $1773 \mathrm{mg} \bullet \mathrm{h} / \mathrm{L}$ [67]. In healthy volunteers, the geometric mean volume of distribution of cefiderocol was $18.0 \mathrm{~L} ; 40-60 \%$ of cefiderocol is bound to plasma proteins, mainly albumin $[67,68]$. Cefiderocol is minimally metabolized and is excreted mainly by the kidneys. Following a single $\left[{ }^{14} \mathrm{C}\right]$-labelled cefiderocol dose of $1 \mathrm{~g}$ infused over $1 \mathrm{~h}$, cefiderocol accounted for $92.3 \%$ of the plasma AUC for total radioactivity, and $98.6 \%$ of total radioactivity was excreted in urine. The estimated geometric mean clearance of cefiderocol is $5.18 \mathrm{~L} / \mathrm{h}$ and the terminal elimination half-life is $2-3 \mathrm{~h}[67,68]$.

Cefiderocol showed good epithelial lining fluid (ELF) penetration in mechanically-ventilated patients with bacterial pneumonia receiving standard-of-care antibacterials [69]. At steady state after multiple 3-h infusions of $2 \mathrm{~g}$ (or kidney function-adjusted dose), geometric mean cefiderocol ELF concentration was $7.63 \mathrm{mg} / \mathrm{L}$ at the end of infusion and $10.4 \mathrm{mg} / \mathrm{L}$ at $2 \mathrm{~h}$ post infusion [69]. In these patients, the ELF to free plasma concentration ratio was 0.212 at the end of infusion and 0.547 at $2 \mathrm{~h}$ post infusion, compared with the ELF to free plasma AUC ratio of 0.239 in healthy subjects, suggesting delayed distribution and sustained exposure of cefiderocol in the ELF in pneumonia patients $[69,70]$.

Kidney function has an impact on cefiderocol pharmacokinetics [71,72]. Cefiderocol dosage should be reduced in patients with moderate (creatinine clearance $\left[\mathrm{CL}_{\mathrm{CR}}\right] \geq 30$ to 
$<60 \mathrm{~mL} / \mathrm{min}$ [68] or $30-59 \mathrm{~mL} / \mathrm{min}$ [67]) or severe $\left(\mathrm{CL}_{\mathrm{CR}}\right.$ $\geq 15$ to $<30 \mathrm{~mL} / \mathrm{min}$ [68] or $15-29 \mathrm{~mL} / \mathrm{min}$ [67]) kidney impairment, or kidney failure $\left(\mathrm{CL}_{\mathrm{CR}}<15 \mathrm{~mL} / \mathrm{min}\right)$, and in those receiving haemodialysis. It should be increased in patients with augmented kidney function $\left(\mathrm{CL}_{\mathrm{CR}} \geq 120\right.$ $\mathrm{mL} / \mathrm{min})[67,68]$. Hepatic impairment is unlikely to affect the pharmacokinetics of cefiderocol [68]. No clinically relevant drug-drug interactions are expected when cefiderocol is coadministered with substrates or inhibitors of CYP enzymes or substrates of drug transporters $[67,68,73]$.

\section{Therapeutic Efficacy of Cefiderocol}

The efficacy of cefiderocol was assessed in two noninferiority trials in patients with cUTI (APEKS-cUTI) [74] or nosocomial pneumonia (APEKS-NP) [75] and in a pathogen-focused trial in patients with serious infections (CREDIBLE-CR) [76]. Design details of these trials are summarized in Table 3. APEKS-cUTI and APEKS-NP excluded patients with known CR bacterial infections, whereas eligible patients in CREDIBLE-CR had to have CR pathogens. Nonetheless, in APEKS-cUTI and APEKS-NP, carbapenem resistance was identified post randomization in several patients. APEKS-NP and CREDIBLE-CR enrolled a high-risk, critically ill patient population. Within each trial, demographic and baseline characteristics were generally similar between the treatment groups and the majority of patients had moderate or severe disease [74-76].

\subsection{In Patients with cUTI}

In the microbiological intent-to-treat (ITT) population $(n=371)$ in APEKS-cUTI, 73\% of patients had cUTI with or without pyelonephritis and $27 \%$ had acute uncomplicated pyelonephritis [74]. Most patients (96\%) had one uropathogen $>1 \times 10^{5} \mathrm{CFU} / \mathrm{mL}$ at baseline, most commonly E. coli $(\approx 63 \%)$ and K. pneumoniae $(20 \%)$; resistance to cefepime or levofloxacin was common among these pathogens [74]. The microbiological ITT population of cUTI in CREDIBLE-CR included 22 patients; the most prevalent $\mathrm{CR}$ pathogens in this population were $K$. pneumoniae $(63 \%)$ and $P$. aeruginosa $(27 \%)$ [76]. The median treatment duration in the cefiderocol and comparator groups were 9 days each in APEKScUTI [74], and 10.5 and 6.5 days in CREDIBLE-CR [76].

In APEKS-cUTI, cefiderocol was noninferior to highdose imipenem/cilastatin for the composite of clinical and microbiological outcomes at test of cure in the microbiological ITT population (Table 4; primary endpoint) [74]. While clinical cure rates were similar between the groups, microbiological eradication rates were significantly higher with cefiderocol (Table 4). Composite outcome results for prespecified subgroups based on age ( $<65$ or $\geq 65$ years), sex (men or women) or clinical diagnosis (cUTI with or without pyelonephritis or acute uncomplicated pyelonephritis) and in subgroups with $E$. coli and $K$. pneumoniae at baseline were consistent with that of the microbiological ITT population. In patients with ESBL-producing uropathogens at baseline, $63 \%$ of 70 cefiderocol and $47 \%$ of 36 imipenem/cilastatin recipients achieved the composite outcome [74].

In CREDIBLE-CR, cefiderocol was associated with favourable microbiological (primary endpoint) and clinical outcomes versus best available therapy (BAT; i.e. a combination of up to three Gram-negative antibacterials) in the microbiological ITT population of cUTI (Table 4) [76].

\subsection{In Patients with Nosocomial Pneumonia}

In the modified ITT population $(n=292)$ in APEKS-NP, $42 \%, 41 \%$ and $17 \%$ of patients had VAP, HAP and healthcare-associated Gram-negative pneumonia (HCAP), respectively [75]. HCAP, which is no longer a separate clinical entity, was included as a subset clinical diagnosis to enrich the study population. The cefiderocol and meropenem groups were well balanced with respect to ventilation status for VAP (98\% vs $98 \%)$ and HAP (37\% vs $35 \%)$

Table 3 Design of randomized, multinational cefiderocol trials in hospitalized adults with aerobic Gram-negative bacterial infections

\begin{tabular}{|c|c|c|c|}
\hline & APEKS-cUTI [74] & APEKS-NP [75] & CREDIBLE-CR [76] \\
\hline Design & $\begin{array}{l}\text { Double-blind, noninferiority, phase } 2 \text {, } \\
\text { US FDA-approved design }\end{array}$ & $\begin{array}{l}\text { Double-blind, noninferiority, phase } 3 \text {, US } \\
\text { FDA-approved design }\end{array}$ & $\begin{array}{l}\text { Open-label, pathogen-focused, descriptive, } \\
\text { phase 3, EMA-approved design }\end{array}$ \\
\hline Treatments $^{\mathrm{a}}$ & $\begin{array}{l}\text { Cefiderocol } 2 \mathrm{~g} \text { q8h } 1 \text {-h infusion or } \\
\text { imipenem/cilastatin } 1 \mathrm{~g} \text { q8h infusion }\end{array}$ & $\begin{array}{l}\text { Cefiderocol } 2 \mathrm{~g} \text { q8h } 3 \text {-h infusion or mero- } \\
\text { penem } 2 \text { g q8h 3-h infusion }\end{array}$ & $\begin{array}{l}\text { Cefiderocol }^{\mathrm{c}} 2 \mathrm{~g} \mathrm{q}^{\mathrm{g}} \mathrm{h} 3 \text {-h infusion or best } \\
\text { available therapy }\end{array}$ \\
\hline
\end{tabular}

$B S I$ bloodstream infection, $c U T I$ complicated urinary tract infection, $H A P$ hospital-acquired pneumonia, $H C A P$ healthcare-associated pneumonia, $p t s$ patients, $q 8 h$ every $8 \mathrm{~h}, V A P$ ventilator-associated pneumonia

${ }^{a}$ Administered for 7-14 days (could be extended up to 21 days in APEKS-NP and CREDIBLE-CR). Dosages adjusted for kidney function; ${ }^{\text {b Both }}$ groups received open-label intravenous linezolid for Gram-positive bacteria and/or methicillin-resistant Staphylococcus aureus coverage; ${ }^{\mathrm{c}}$ One adjunctive agent (excluding polymyxins, cephalosporins and carbapenems) was permitted in pts with pneumonia or BSI/sepsis; ${ }^{\mathrm{d} A}$ maximum of three prespecified antibacterials, dosed as per the country's label 
Table 4 Efficacy of cefiderocol in patients with Gram-negative bacterial infections in clinical studies

Treatment (no. of $\mathrm{pts}^{\mathrm{a}}$ ) ACM at day $14 \quad$ Clinical cure at TOC

$(\% \mathrm{pts})[95 \% \mathrm{CI}] \quad \quad(\% \mathrm{pts})[95 \% \mathrm{CI}] \quad \mathrm{TOC}^{\mathrm{b}}(\% \mathrm{pts})[95 \% \mathrm{CI}]$

\begin{tabular}{|c|c|c|c|}
\hline In pts with complicated urinary & & & \\
\hline APEKS-cUTI [74] & & & \\
\hline Cefiderocol $(252)^{\mathrm{c}}$ & ND & 90 & 73 \\
\hline Imipenem/cilastatin (119) & ND & 87 & 56 \\
\hline Treatment difference & ND & $2.39[-4.66$ to 9.44$]$ & $17.25[6.92-27.58]$ \\
\hline CREDIBLE-CR [76] & & & \\
\hline Cefiderocol (17) & $12[1.5-36.4]$ & $71[44.0-89 \cdot 7]$ & $53^{\mathrm{d}}[27.8-77.0]$ \\
\hline Best available therapy (5) & $40[5.3-85.3]$ & 60 [14.7-94.7] & $20^{\mathrm{d}}[0.5-71.6]$ \\
\hline In pts with nosocomial pneumo & & & \\
\hline APEKS-NP [75] & & & \\
\hline Cefiderocol (145] & 12.4 & 65 & 41 \\
\hline Meropenem (147) & 11.6 & 67 & 42 \\
\hline Treatment difference & $0.8^{*}[-6.6 \text { to } 8.2]^{\mathrm{d}}$ & $-1.8[-12.7$ to 9.0$]$ & $-0.8[-12.1$ to 10.5$]$ \\
\hline CREDIBLE-CR [76] & & & \\
\hline Cefiderocol (40) & $25[12.7-41.2]$ & $50^{\mathrm{d}}[33.8-66.2]$ & $23[10.8-38.5]$ \\
\hline Best available therapy (19) & $11[1.3-43.7]$ & $53^{\mathrm{d}}[28.9-75.6]$ & $21[6.1-45.6]$ \\
\hline In pts with bloodstream infectic & R) $[76]$ & & \\
\hline Cefiderocol (23) & $22[7.5-43.7]$ & $43^{\mathrm{d}}[23.2-65.5]$ & 30 [13.2-52.9] \\
\hline Best available therapy (14) & $7[0.2-33.9]$ & $43^{\mathrm{d}}[17.7-71.1]$ & $29[8.4-58.1]$ \\
\hline In overall population with $\mathrm{CR} i$ & {$[76]$} & & \\
\hline Cefiderocol $(80)$ & $21[12.9-31.8]$ & $53[41.0-63.8]$ & $31[21.3-42.6]$ \\
\hline Best available therapy (38) & $13[4.4-28.1]$ & $50[33.4-66.6]$ & $24[11.4-40.2]$ \\
\hline
\end{tabular}

$A C M$ all-cause mortality, ITT intent-to-treat, $N D$ not determined, pts patients, TOC test of cure ${ }^{*} p=0.002$ for noninferiority (at $12.5 \%$ margin) hypothesis

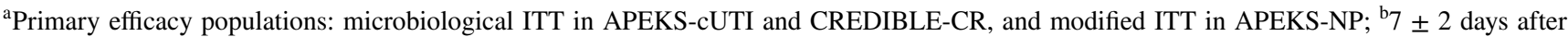
the end of treatment; ${ }^{\circ}$ Cefiderocol was noninferior (at 15\% margin) to the comparator for the primary endpoint of composite of clinical and microbiological outcomes at TOC (73\% vs 55\%; treatment difference $18.58 \%$; $95 \%$ CI $8.23-28.92 ; p=0.0004)$; ${ }^{\mathrm{d}}$ Primary endpoint

subgroups, empirical treatment failure (33\% vs 32\%), presence in an ICU at randomization (70\% vs 66\%). Among HCAP patients, more cefiderocol than meropenem recipients required mechanical ventilation at baseline (33\% vs $9 \%)$. The two groups were also similar with respect to kidney function, Acute Physiology and Chronic Health Evaluation (APACHE) II score, Clinical Pulmonary Infection Score (CPIS) and Sequential Organ Failure Assessment (SOFA) score. A qualifying Gram-negative pathogen was identified in 251 (86\%) patients, with K. pneumoniae (32\%), P. aeruginosa (16\%), A. baumannii (16\%) and E. coli (14\%) the most prevalent. The mean duration of study treatment was 10.4 and 10.1 days [75].

In APEKS-NP, cefiderocol was noninferior to high-dose, extended-infusion meropenem for all-cause mortality at day 14 in the modified ITT population (Table 4; primary endpoint) [75]. No clinically relevant between-group differences were seen for this outcome in prespecified subgroups based on clinical diagnosis, sex, race, geographical region, APACHE II score, CPIS, concomitant bacteraemia, kidney function, empirical treatment failure, ICU or ventilation status at randomization, or baseline pathogens. All-cause mortality at day 28 was also similar between cefiderocol and meropenem groups in the modified ITT population $(21.0 \%$ vs $20.5 \%$ ) and in subgroups based on higher risk scores (e.g. APACHE II score $\geq 20$; SOFA score $\geq 7$ ), previous empirical treatment failure or ICU status at randomization [75].

Clinical and microbiological outcomes were also similar between cefiderocol and meropenem groups in the modified ITT population in APEKS-NP (Table 4) and in subgroups based on the five most prevalent baseline pathogens [75]. In patients with HCAP, clinical cure rates were $82 \%$ and $91 \%$. Clinical outcome data are generally supported by change from baseline in CPIS and SOFA score [75].

During treatment in APEKS-NP, six patients in the cefiderocol group and five patients in the meropenem group had $a \geq 4$-fold increase in the MIC values for respective agents; none of these patients died by day 14 [75]. The isolates with increased MIC values were: $K$. pneumoniae (3 patients), $E$. aerogenes (2) and E. cloacae and S. marcescens (1) in the 
cefiderocol group; $K$. pneumoniae (1), P. aeruginosa (3) and $C$. freundii (1) in the meropenem group. Despite the increase, cefiderocol MIC values remained at $\leq 1 \mathrm{mg} / \mathrm{L}$ for all but one isolate (E. cloacae; MIC $4 \mu \mathrm{g} / \mathrm{mL}$ in one patient) at treatment end [75].

In CREDIBLE-CR, clinical cure (primary endpoint) and microbiological eradication rates in the microbiological ITT population of nosocomial pneumonia $(n=118)$ were comparable between cefiderocol and BAT groups (Table 4) [76]. In this population, the most prevalent $\mathrm{CR}$ pathogens at baseline in the cefiderocol group were A. baumannii (65\% vs $53 \%$ in the BAT group), $P$. aeruginosa (15\% vs $26 \%), K$. pneumoniae (15\% vs $26 \%)$ and S. maltophilia (13\% vs 0\%) [76].

\subsection{In Patients with BSI/sepsis}

In CREDIBLE-CR, cefiderocol was comparable to BAT with regards to clinical cure (primary endpoint) and microbiological eradication rates in the CR microbiological ITT population of BSI/sepsis $(n=37)$ (Table 4) [76]. The most prevalent CR pathogens in the cefiderocol and BAT groups in this population were K. pneumoniae (44\% vs $29 \%$ ), A. baumannii (44\% vs 50\%) and P. aeruginosa (9\% vs 21\%) [76].

\subsection{In Patients with CR Bacterial Infections}

In the overall CREDIBLE-CR population, cefiderocol showed comparable clinical and microbiological efficacy to BAT (Table 4), with numerical between-group differences favouring cefiderocol in patients with infections caused by Enterobacterales (clinical cure 66\% vs 45\%; microbiological eradication $48 \%$ vs $18 \%$ ) or metallo- $\beta$-lactamase producers (75\% vs $29 \%$; $44 \%$ vs $14 \%$ ) [76]. A similar proportion of patients in the respective treatment groups achieved the composite endpoint of survival without the need to change antibacterial(s) due to toxicity or absence of efficacy (63\% vs $61 \%$; treatment difference $1.1 \%$; $95 \%$ CI -17.7 to 20.0) [76].

In CREDIBLE-CR, the all-cause mortality rate in the CR microbiological ITT population was higher in the cefiderocol than the BAT group at day 14 (Table 4). Similar result was seen in the safety population at day $14(19 \%$ vs $12 \%)$, day 28 ( $25 \%$ vs $18 \%$ ) and at study end (34\% vs $18 \%)$; the between-group difference was particularly evident in patients with nosocomial pneumonia or BSI/sepsis, caused by Acinetobacter spp. with or without co-infection with another pathogen [76]. Conversely, in patients with infections due to nonfermenters other than Acinetobacter spp., mortality was not higher with cefiderocol versus BAT [68]. The cause of the increased mortality in the cefiderocol group has not been established [68], although, among Acinetobacter-infected patients, baseline mortality risk (septic shock and ICU status) was higher in the cefiderocol group than in the BAT group [76].

After randomization in APEKS-NP, 30 patients in the cefiderocol and 26 in the meropenem group were found to have pathogens with a meropenem MIC of $>8 \mu \mathrm{g} / \mathrm{mL}$ at baseline [75]. Clinical and microbiological outcomes in this subgroup were less favourable compared with those with MIC $<8 \mu \mathrm{g} / \mathrm{mL}$. In the MIC $>8 \mu \mathrm{g} / \mathrm{mL}$ subgroup, all-cause mortality rates in the cefiderocol and meropenem groups were $20 \%$ and $19 \%$ at day $14(27 \%$ and $31 \%$ at day 28$)$; at test of cure, clinical cure rates were $57 \%$ and $58 \%$, and microbiological eradication rates were $40 \%$ and $31 \%$ [75].

\section{Safety and Tolerability of Cefiderocol}

Cefiderocol was generally well tolerated in patients with cUTI in APEKS-cUTI [74] and had an acceptable tolerability profile in patients with nosocomial pneumonia in APEKS-NP [75] and those with CR serious infections in CREDIBLE-CR [76]. Apart from the unexplained imbalance in all-cause mortality in CREDIBLE-CR (Sect. 4.4), the safety profile of cefiderocol was consistent with that expected for a cephalosporin, with no new safety signals seen in these clinical trials [74-76]. The adverse event (AE) profile of cefiderocol was generally similar to that of comparators [74-77].

The most common adverse reactions with cefiderocol were diarrhoea, infusion site reactions, constipation, rash, candidiasis, cough, elevations in liver tests, headache, hypokalaemia, nausea and vomiting in patients with cUTI, and elevations in liver function tests, hypokalaemia, diarrhoea, hypomagnesemia and atrial fibrillation in patients with nosocomial pneumonia [67].

Treatment-emergent AEs, severe AEs and serious AEs occurred in similar proportions of patients in each treatment arm in APEKS-cUTI [74], APEKS-NP [75] and CREDIBLE-CR [76]. Across these trials, treatmentrelated AEs (TRAEs) occurred in $10.2 \%$ of 549 cefiderocol recipients, $<1 \%$ had serious TRAEs and $1.5 \%$ discontinued treatment because of TRAEs [77]. The most common TRAEs with cefiderocol were elevations in liver function tests and diarrhoea [77]. In CREDIBLE-CR, treatmentemergent AEs led to more deaths in the cefiderocol arm than in the BAT arm (33.7\% vs $18.4 \%)$, particularly infections and infestations $(20.8 \%$ vs $6.1 \%)$ and septic shock (10.9\% vs. $6.1 \%)$ [10].

Consistent with the known class effects of cephalosporins, AEs of special interest with cefiderocol include Clostridium difficile-related AEs, liver-related AEs, seizures and hypersensitivity reactions [77]. Across all trials, C. difficile-related AEs (mostly mild or moderate) occurred in eight cefiderocol recipients, including one serious TRAE 
of colitis. The incidence of liver-related AEs was similar between treatment groups in APEKS-cUTI ( $0.7 \%$ with cefiderocol vs $0.7 \%$ with imipenem/cilastatin) and APEKS-NP ( $25 \%$ with cefiderocol vs $24 \%$ with meropenem), but was higher with cefiderocol in CREDIBLE-CR $(29.7 \%$ vs $14.3 \%$ with BAT). The majority of liver-related AEs in CREDIBLE-CR had an alternate aetiology (underlying medical history or concomitant medication) and none met the criteria for Hy's law or drug-induced liver injury. Four cefiderocol recipients had seizures and none were treatment-related. The incidence of rash or hypersensitivity reactions was similar between cefiderocol and comparator groups and none were serious; cefiderocol-related rash occurred in one patient each in APEKS-NP and CREDIBLE-CR [77].

As cefiderocol is a siderophore antibacterial, its effect on host iron homeostasis is of interest. In APEKS-cUTI, APEKS-NP and CREDIBLE-CR, it did not increase the risk of iron homeostasis-related AEs compared with nonsiderophore antibacterials [77].

\section{Dosage and Administration of Cefiderocol}

Cefiderocol is approved for the treatment of adults with cUTIs (including pyelonephritis), HAP or VAP, caused by designated susceptible Gram-negative bacteria, in the USA [67] and for the treatment of infections caused by aerobic Gram-negative bacteria in adults with limited treatment options in the EU [68]. The recommended dosage of cefiderocol in patients with normal kidney function is $2 \mathrm{~g}$ every $8 \mathrm{~h}$ (3-h infusion) for 5-14 days [67, 68]. As cefiderocol is predominantly excreted by the kidneys (Sect. 3), dosage adjustment is recommended for patients with impaired or augmented kidney function and in those with kidney failure or who require dialysis $[67,68]$. Consult local prescribing information for detailed information, including dosage and administration details, specific dosage adjustments for kidney function, contraindications, potential drug interactions, use in special patient populations, and warnings and precautions.

\section{Place of Cefiderocol in Managing Serious Gram-Negative Bacterial Infections}

$\beta$-Lactams are the mainstay of treatment for Gram-negative bacterial infections; against $\beta$-lactam-resistant pathogens, conventional options are colistin, fosfomycin and tigecycline, all of which have efficacy and/or toxicity limitations [10]. Cefiderocol is the first siderophore cephalosporin against Gram-negative bacteria to be approved in the USA [67] and the EU [68]. The approved indications vary between the USA and the EU (Sect. 6). Other approved new agents for cUTI, HAP or VAP include ceftazidime-avibactam, ceftozolane-tazobactam, imipenem/ cilastatin-relebactam, meropenem-vaborbactam and plazomicin. In the USA, cefiderocol, ceftozolane-tazobactam, ceftazidime-avibactam and imipenem/cilastatin-relebactam are approved for cUTI as well as for HAP and VAP in adults; ceftazidime-avibactam is also approved for cUTI in children.

Cefiderocol has a unique mechanism of bacterial cell entry and a wide spectra of Gram-negative antibacterial activity (Sect. 2); of the available new agents, only cefiderocol is stable against all classes of $\beta$-lactamases, including metallo- $\beta$-lactamases $[8,9]$. A differentiating feature of cefiderocol is its activity against MDR $P$. aeruginosa, MDR A. baumannii, S. maltophilia and B. cepacia [12].

The choice of antibacterial for cUTI depends on a number of factors, including disease severity and local resistance patterns [78]. The initial empirical therapy should be followed by definitive antibacterial therapy based on the uropathogen identified [78]. Cefiderocol is approved for cUTI (including pyelonephritis) caused by E. coli, K. pneumoniae, P. mirabilis, $P$. aeruginosa and E. cloacae complex [67]. The approval was based on results from the APEKS-cUTI trial in which cefiderocol was noninferior to imipenem/cilastatin for the treatment of cUTI (Sect. 4.1). Furthermore, a network meta-analysis (5 studies, 2349 patients), subject to its limitations such as inclusion of studies with different designs, found that cefiderocol was superior to imipenem/cilastatin for microbiological eradication and was similar to ceftazidime-avibactam, ceftozolane-tazobactam, doripenem and levofloxacin for clinical or microbiological outcomes [79].

According to IDSA, cefiderocol is one of the preferred treatment options for cUTI and pyelonephritis caused by CR Enterobacterales (resistant to both ertapenem and meropenem) or by difficult-to-treat resistant $P$. aeruginosa [80]. Cefiderocol also has a place in the management of infections outside of the urinary tract caused by CR Enterobacterales [preferred option against NDM and other metallo- $\beta$-lactamase-producing strains, and an alternative option when carbapenemase (including KPC) testing results are either not available or negative]. The increased mortality seen with cefiderocol versus BAT in CREDIBLE-CR does not appear to extend to patients with UTIs [80].

The choice of empiric antibacterial therapy for HAP and VAP is guided by the risk for MDR pathogens and mortality, and in high-risk patients by the presence or absence of septic shock [81, 82]. The choice of agent(s) for definitive therapy is based on antimicrobial susceptibility testing results and patient-specific factors (e.g. allergy, comorbidities) [81, 82]. Cefiderocol is approved for HAP and VAP caused by A. baumannii complex, E. coli, E. cloacae 
complex, K. pneumoniae, $P$. aeruginosa and $S$. marcescens [67]. In the registrational APEKS-NP trial, cefiderocol was noninferior to meropenem for 14-day all-cause mortality in critically ill patients with nosocomial pneumonia caused by Gram-negative bacteria, including those at high risk of MDR pathogens (Sect. 4.2). Of note, APEKS-NP is the first study to include extended infusion of high-dose meropenem ( $2 \mathrm{~g}$ every $8 \mathrm{~h}$ ) as a comparator. The definitive therapy options recommended by the current 2016 IDSA and the American Thoracic Society guidelines include carbapenem, ampicillin/ sulbactam and polymyxins against Acinetobacter spp. and polymyxins against CR pathogens [82]. The approval of cefiderocol is too recent for the drug to have been included in these guidelines.

Carbapenem resistance is a good indicator of multidrug resistance and is associated with limited treatment options [83]. In the pathogen-focused CREDIBLE-CR trial, cefiderocol had similar clinical and microbiological outcomes to BAT (up to three drugs) in a heterogeneous patient population with CR infections (Sect. 4.4). The increased mortality in the cefiderocol arm in this trial remains unexplained. While CREDIBLE-CR represents a real-world setting, it was a small open-label study and included only a few patients with complicated intra-abdominal infections [76]. Based on the CREDIBLE-CR data, cefiderocol was approved in the EU for the treatment of Gram-negative infections in adults who have limited treatment options [68]. The only other drug approved for this indication is imipenem/cilastatin-relebactam, which has no in vitro activity against class B (e.g. NDM, VIM) and D (e.g. OXA-48) carbapenemases [84]. According to NICE, cefiderocol may be an option for treating Gram-negative infections in adults who have limited treatment options, particularly when other antimicrobials have failed [83].

Cefiderocol was well tolerated in patients with cUTI and had an acceptable tolerability profile in critically ill patients with HAP, VAP or life-threatening CR infections (Sect. 5). The AE and safety profile of cefiderocol was broadly similar to those of other $\beta$-lactam agents. The increased mortality seen with cefiderocol in patients with CR infections was not due to cefiderocol-related toxicity.

Limited real-world data suggest that cefiderocol monotherapy or in combination with other antibacterials may be useful as rescue treatment for severe, CR or extensivelyresistant, Gram-negative infections in critically ill or immunocompromised patients $[85,86]$. According to a systematic review ( 24 case series/reports; 60 patients), cefiderocol was effective and well tolerated in managing difficult-to-treat CR infections in the real-world setting (clinical cure rate $80 \%$, microbiological eradication rate $41.7 \%$, all-cause mortality $20 \%$, AE incidence $13.3 \%$ ) [87].

In conclusion, cefiderocol is a siderophore cephalosporin with activity against CR and MDR Gram-negative bacteria; it is stable against all four Ambler classes of $\beta$-lactamases. The drug was effective and had a good tolerability and safety profile in patients with cUTI, HAP, VAP or CR serious infections due to aerobic Gram-negative bacteria. Further data on real-world experience, resistance development and cost-effectiveness of cefiderocol would be of interest. More data are also required to clarify the increased mortality seen with cefiderocol in CREDIBLE-CR. Current evidence indicates that cefiderocol is a novel, emerging, definitive therapy option that is a useful addition to the current treatment options for adults with susceptible Gram-negative bacterial infections (including cUTI, HAP or VAP) for whom there are limited treatment options.

Data Selection Cefiderocol: 411 records identified

Search Strategy: EMBASE, MEDLINE and PubMed from 1946 to present. Clinical trial registries/databases and websites were also searched for relevant data. Key words were Cefiderocol, Fetcroja and Fetroja. Records were limited to those in English language. Searches last updated 21 July 2021.

Supplementary Information The online version contains supplementary material available at https://doi.org/10.1007/s40265-021-01580-4.

Acknowledgements During the peer review process the manufacturer of cefiderocol was also offered an opportunity to review this article. Changes resulting from comments received were made on the basis of scientific and editorial merit.

\section{Declarations}

Funding The preparation of this review was not supported by any external funding.

Authorship and Conflict of Interest Yahiya Syed is a salaried employee of Adis International Ltd/Springer Nature, and declares no relevant conflicts of interest. All authors contributed to the review and are responsible for the article content.

Ethics approval, Consent to participate, Consent to publish, Availability of data and material, Code availability Not applicable.

Open Access This article is licensed under a Creative Commons Attribution-NonCommercial 4.0 International License, which permits any non-commercial use, sharing, adaptation, distribution and reproduction in any medium or format, as long as you give appropriate credit to the original author(s) and the source, provide a link to the Creative Commons licence, and indicate if changes were made. The images or other third party material in this article are included in the article's Creative Commons licence, unless indicated otherwise in a credit line to the material. If material is not included in the article's Creative Commons licence and your intended use is not permitted by statutory regulation or exceeds the permitted use, you will need to obtain permission directly from the copyright holder. To view a copy of this licence, visit http://creativecommons.org/licenses/by-nc/4.0/. 


\section{References}

1. Golan Y. Empiric therapy for hospital-acquired, Gram-negative complicated intra-abdominal infection and complicated urinary tract infections: a systematic literature review of current and emerging treatment options. BMC Infect Dis. 2015. https://doi. org/10.1186/s12879-015-1054-1.

2. Jean SS, Chang YC, Lin WC, et al. Epidemiology, treatment, and prevention of nosocomial bacterial pneumonia. J Clin Med. 2020. https://doi.org/10.3390/jcm9010275.

3. Diekema DJ, Hsueh PR, Mendes RE, et al. The microbiology of bloodstream infection: 20-year trends from the SENTRY antimicrobial surveillance program. Antimicrob Agents Chemother. 2019;63(7):e00355-e419.

4. World Health Organization. Global priority list of antibioticresistant bacteria to guide research, discovery, and development of new antibiotics. 2017. https://www.who.int. Accessed 11 May 2021.

5. Centers for Disease Control and Prevention. Antibiotic resistance threats in the United States. 2019. https://www.cdc.gov. Accessed 11 May 2021.

6. Brooke JS. Stenotrophomonas maltophilia: an emerging global opportunistic pathogen. Clin Microbiol Rev. 2012;25(1):2-41.

7. Codjoe FS, Donkor ES. Carbapenem resistance: a review. Med Sci (Basel). 2017. https://doi.org/10.3390/medsci6010001.

8. Jean SS, Gould IM, Lee WS, et al. New drugs for multidrugresistant Gram-negative organisms: time for stewardship. Drugs. 2019;79(7):705-14.

9. Doi Y. Treatment options for carbapenem-resistant gram-negative bacterial infections. Clin Infect Dis. 2019;69(Suppl 7):S565-75.

10. European Medicines Agency. Fetcroja: assessment report. 2020. https://www.ema.europa.eu. Accessed 11 May 2021.

11. Sato T, Yamawaki K. Cefiderocol: discovery, chemistry, and in vivo profiles of a novel siderophore cephalosporin. Clin Infect Dis. 2019;69(Suppl 7):S538-43.

12. Zhanel GG, Golden AR, Zelenitsky S, et al. Cefiderocol: a siderophore cephalosporin with activity against carbapenemresistant and multidrug-resistant Gram-negative bacilli. Drugs. 2019;79(3):271-89.

13. Ito A, Nishikawa T, Matsumoto S, et al. Siderophore cephalosporin cefiderocol utilizes ferric iron transporter systems for antibacterial activity against Pseudomonas aeruginosa. Antimicrob Agents Chemother. 2016;60(12):7396-401.

14. Ito $\mathrm{A}$, Sato $\mathrm{T}$, Ota $\mathrm{M}$, et al. In vitro antibacterial properties of cefiderocol, a novel siderophore cephalosporin, against Gram-negative bacteria. Antimicrob Agents Chemother. 2018;62(1):e01454-e1517.

15. Aoki T, Yoshizawa H, Yamawaki K, et al. Cefiderocol (S-649266), a new siderophore cephalosporin exhibiting potent activities against Pseudomonas aeruginosa and other Gram-negative pathogens including multi-drug resistant bacteria: structure activity relationship. Eur J Med Chem. 2018;155:847-68.

16. Ito-Horiyama T, Ishii Y, Ito A, et al. Stability of novel siderophore cephalosporin S-649266 against clinically relevant carbapenemases. Antimicrob Agents Chemother. 2016;60(7):4384-6.

17. Iregui $A$, Khan $Z$, Landman $D$, et al. Activity of cefiderocol against Enterobacterales, Pseudomonas aeruginosa, and Acinetobacter baumannii endemic to medical centers in New York City. Microb Drug Resist. 2020;26(7):722-6.

18. Kazmierczak KM, Tsuji M, Wise MG, et al. In vitro activity of cefiderocol, a siderophore cephalosporin, against a recent collection of clinically relevant carbapenem-non-susceptible Gramnegative bacilli, including serine carbapenemase- and metallo$\beta$-lactamase-producing isolates (SIDERO-WT-2014 study). Int J Antimicrob Agents. 2019;53(2):177-84.
19. Clinical and Laboratory Standards Institute. CLSI Winter 2021 Susceptibility Testing Meeting Series. 2021. https://clsi.org/umbra co/api/astfiles/download/?id=43239. Accessed 4 Jun 2021.

20. Morris CP, Bergman Y, Tekle T, et al. Cefiderocol antimicrobial susceptibility testing against multidrug-resistant Gram-negative bacilli: a comparison of disk diffusion to broth microdilution. J Clin Microbiol. 2020;59(1):e01649-e1720.

21. Yamano Y, Takemura M, Longshaw $\mathrm{C}$, et al. Differences in interpretative breakpoints between CLSI, FDA and EUCAST impact reporting of susceptibility and resistance to cefiderocol [abstract no. 1269]. Open Forum Infect Dis. 2020;7(Suppl 1):S651.

22. Hackel MA, Tsuji M, Yamano Y, et al. In vitro activity of the siderophore cephalosporin, cefiderocol, against a recent collection of clinically relevant gram-negative bacilli from North America and Europe, including carbapenem-nonsusceptible isolates (SIDERO-WT-2014 study). Antimicrob Agents Chemother. 2017;61(9):e00093-e117.

23. Karlowsky JA, Hackel MA, Tsuji M, et al. In vitro activity of cefiderocol, a siderophore cephalosporin, against Gram-negative bacilli isolated by clinical laboratories in North America and Europe in 2015-2016: SIDERO-WT-2015. Int J Antimicrob Agents. 2019;53(4):456-66.

24. Longshaw $\mathrm{C}$, Tsuji M, Hackel MM, et al. In vitro activity of cefiderocol (CFDC), a novel siderophore cephalosporin, against difficult-to-treat-resistant (DTR) gram-negative bacterial pathogens from the multi-national sentinel surveillance study, SIDEROWT (2014-2017) [abstract no. 679]. Open Forum Infect Dis. 2019;6(Suppl 2):S309-10.

25. Ukai $Y$, Nakamura R, Oota $M$, et al. In vitro antibacterial activity of cefiderocol against non-fermenter clinical strains collected in North America and Europe from multinational surveillance studies SIDERO-WT-2014-2018 [abstract no. 164]. Open Forum Infect Dis. 2020;7(Suppl 1):S211-2.

26. Tsuji M, Hackel M, Echols R, et al. In vitro antibacterial activity of cefiderocol against Gram-negative clinical strains collected in North America and Europe, SIDERO-WT-2016 [abstract no. AAR-767 plus poster]. In: American Society of Microbiology Annual Meeting 2019.

27. Hackel MA, Tsuji M, Yamano Y, et al. In vitro activity of the siderophore cephalosporin, cefiderocol, against carbapenemnonsusceptible and multidrug-resistant isolates of Gram-negative bacilli collected worldwide in 2014 to 2016. Antimicrob Agents Chemother. 2018;62(2): e01968-e2017.

28. Johnston BD, Thuras P, Porter SB, et al. Activity of cefiderocol, ceftazidime-avibactam, and eravacycline against carbapenemresistant Escherichia coli isolates from the United States and international sites in relation to clonal background, resistance genes, co-resistance, and region. Antimicrob Agents Chemother. 2020;64(10):e00797-e820.

29. Kohira N, West J, Ito A, et al. In vitro antimicrobial activity of a siderophore cephalosporin, S-649266, against Enterobacteriaceae clinical isolates, including carbapenem-resistant strains. Antimicrob Agents Chemother. 2016;60(2):729-34.

30. Albano M, Karau MJ, Schuetz AN, et al. Comparison of agar dilution to broth microdilution for testing in vitro activity of cefiderocol against Gram-negative bacilli. J Clin Microbiol. 2020;59(1):e00966-e1020.

31. Golden AR, Adam HJ, Baxter M, et al. In vitro activity of cefiderocol, a novel siderophore cephalosporin, against Gram-negative bacilli isolated from patients in Canadian intensive care units. Diagn Microbiol Infect Dis. 2020. https://doi.org/10.1016/j.diagm icrobio.2020.115012.

32. Kresken M, Korte-Berwanger M, Gatermann SG, et al. In vitro activity of cefiderocol against aerobic Gram-negative bacterial pathogens from Germany. Int J Antimicrob Agents. 2020. https:// doi.org/10.1016/j.ijantimicag.2020.106128. 
33. Falagas ME, Skalidis T, Vardakas KZ, et al. Activity of cefiderocol (S-649266) against carbapenem-resistant Gram-negative bacteria collected from inpatients in Greek hospitals. J Antimicrob Chemother. 2017;72(6):1704-8.

34. Matsumoto S, Kanazawa S, Sato T, et al. Activities of cefiderocol with simulated human plasma concentrations against carbapenemresistant Gram-negative bacilli in an in vitro chemostat model. Antimicrob Agents Chemother. 2020;64(11):e01128-e1220.

35. Kanazawa S, Sato T, Kohira N, et al. Susceptibility of imipenem-susceptible but meropenem-resistant bla(IMP-6)-carrying Enterobacteriaceae to various antibacterials, including the siderophore cephalosporin cefiderocol. Antimicrob Agents Chemother. 2017;61(7):e00576-e617.

36. Delgado-Valverde M, Conejo MDC, Serrano L, et al. Activity of cefiderocol against high-risk clones of multidrug-resistant Enterobacterales, Acinetobacter baumannii, Pseudomonas aeruginosa and Stenotrophomonas maltophilia. J Antimicrob Chemother. 2020;75(7):1840-9.

37. Hsueh SC, Lee YJ, Huang YT, et al. In vitro activities of cefiderocol, ceftolozane/tazobactam, ceftazidime/avibactam and other comparative drugs against imipenem-resistant Pseudomonas aeruginosa and Acinetobacter baumannii, and Stenotrophomonas maltophilia, all associated with bloodstream infections in Taiwan. J Antimicrob Chemother. 2019;74(2):380-6.

38. Mushtaq S, Sadouki Z, Vickers A, et al. In vitro activity of cefiderocol, a siderophore-cephalosporin, against multidrug-resistant Gram-negative bacteria. Antimicrob Agents Chemother. 2020;64(12):e01582-e1620.

39. Biagi M, Vialichka A, Jurkovic M, et al. Activity of cefiderocol alone and in combination with levofloxacin, minocycline, polymyxin $b$, or trimethoprim-sulfamethoxazole against multidrugresistant Stenotrophomonas maltophilia. Antimicrob Agents Chemother. 2020;64(9):e00559-e620.

40. Rolston KVI, Gerges B, Shelburne S, et al. Activity of cefiderocol and comparators against isolates from cancer patients. Antimicrob Agents Chemother. 2020;64(5):e01955-e2019.

41. Jacobs MR, Abdelhamed AM, Good CE, et al. ARGONAUT-I: activity of cefiderocol (S-649266), a siderophore cephalosporin, against Gram-negative bacteria, including carbapenem-resistant nonfermenters and Enterobacteriaceae with defined extendedspectrum $\beta$-lactamases and carbapenemases. Antimicrob Agents Chemother. 2019;63(1):e01801-e1818.

42. Ito A, Kohira N, Bouchillon SK, et al. In vitro antimicrobial activity of S-649266, a catechol-substituted siderophore cephalosporin, when tested against non-fermenting Gram-negative bacteria. J Antimicrob Chemother. 2016;71(3):670-7.

43. Yamano Y. In vitro activity of cefiderocol against a broad range of clinically important Gram-negative bacteria. Clin Infect Dis. 2019;69(Suppl 7):S544-51.

44. Takemura M, Kazmierczak KM, Hackel M, et al. In vitro activity of cefiderocol against metallo $\beta$-lactamase-producing Gramnegative bacteria in North America and Europe between 2014 and 2017: SIDERO-WT-2014 to -2016 studies [abstract no. 1252]. Open Forum Infect Dis. 2020;7(Suppl 1):S643-4.

45. Tsuji M, Kazmierczak KM, Hackel M, et al. Cefiderocol (S649266) susceptibility against globally isolated meropenem non-susceptible Gram-negative bacteria containing serine- and metallo-carbapenemase genes [abstract no. 622 plus poster]. In: American Society of Microbiology Annual Meeting 2018.

46. Shields RK, Kline EG, Jones CE, et al. Cefiderocol minimum inhibitory concentrations against ceftazidime-avibactam susceptible and resistant carbapenem-resistant Enterobacteriaceae [abstract no. 620 plus poster]. In: American Society of Microbiology Annual Meeting. 2018.

47. Ito A, Nishikawa T, Ota M, et al. Stability and low induction propensity of cefiderocol against chromosomal AmpC $\beta$-lactamases of Pseudomonas aeruginosa and Enterobacter cloacae. J Antimicrob Chemother. 2018;73(11):3049-52.

48. Dobias J, Dénervaud-Tendon V, Poirel L, et al. Activity of the novel siderophore cephalosporin cefiderocol against multidrugresistant Gram-negative pathogens. Eur J Clin Microbiol Infect Dis. 2017;36(12):2319-27.

49. Kidd JM, Abdelraouf K, Nicolau DP. Efficacy of humanized cefiderocol exposure is unaltered by host iron overload in the thigh infection model. Antimicrob Agents Chemother. 2019;64(1):e01767-e1819.

50. Chen IH, Kidd JM, Abdelraouf K, et al. Comparative in vivo antibacterial activity of human-simulated exposures of cefiderocol and ceftazidime against Stenotrophomonas maltophilia in the murine thigh model. Antimicrob Agents Chemother. 2019;63(12):e01558-e1619.

51. Nakamura R, Ito-Horiyama T, Takemura M, et al. In vivo pharmacodynamic study of cefiderocol, a novel parenteral siderophore cephalosporin, in murine thigh and lung infection models. Antimicrob Agents Chemother. 2019;63(9):e02031-e2118.

52. Stainton SM, Monogue ML, Tsuji M, et al. Efficacy of humanized cefiderocol exposures over 72 hours against a diverse group of Gram-negative isolates in the neutropenic murine thigh infection model. Antimicrob Agents Chemother. 2019;63(2):e01040-e1118.

53. Ghazi IM, Monogue ML, Tsuji M, et al. Humanized exposures of cefiderocol, a siderophore cephalosporin, display sustained in vivo activity against siderophore-resistant $P$ seudomonas aeruginosa. Pharmacology. 2018;101(5-6):278-84.

54. Ghazi IM, Monogue ML, Tsuji M, et al. Pharmacodynamics of cefiderocol, a novel siderophore cephalosporin, in a Pseudomonas aeruginosa neutropenic murine thigh model. Int J Antimicrob Agents. 2018;51(2):206-12.

55. Monogue ML, Tsuji M, Yamano Y, et al. Efficacy of humanized exposures of cefiderocol (S-649266) against a diverse population of Gram-negative bacteria in a murine thigh infection model. Antimicrob Agents Chemother. 2017;61(11):e01022-e1117.

56. Nakamura R, Oota M, Yoshitomi T, et al. In vitro and in vivo antimicrobial activity of cefiderocol and comparators against Stenotrophomonas maltophilia [abstract no. 2577]. In: 30th European Congress of Clinical Microbiology and Infectious Diseases. 2020.

57. Matsumoto S, Singley CM, Hoover J, et al. Efficacy of cefiderocol against carbapenem-resistant Gram-negative bacilli in immunocompetent-rat respiratory tract infection models recreating human plasma pharmacokinetics. Antimicrob Agents Chemother. 2017;61(9):e00700-e717.

58. Ota K, Kaku N, Uno N, et al. Efficacy of cefiderocol against carbapenem-resistant $A$. baumannii and $P$. aeruginosa in ventilatorassociated pneumonia mouse model [abstract no. 1273]. Open Forum Infect Dis. 2020;7(Suppl 1):S653.

59. Yamano Y, Takemura M, Kazmierczak KM, et al. Molecular profile of $\beta$-lactamase genes and siderophore-dependent iron transporter genes of cefiderocol high MIC isolates from SIDERO-WT studies [abstract no. 1452]. Open Forum Infect Dis. 2020;7(Suppl 1):S728-9.

60. Ito A, Hackel M, Sahm D, et al. Characterization of isolates showing high MICs to cefiderocol from global surveillance study SIDERO-CR-2014/2016 [abstract no. 1857]. In: 29th European Congress of Clinical Microbiology and Infectious Diseases. 2019.

61. Malik S, Kaminski M, Landman D, et al. Cefiderocol resistance in Acinetobacter baumannii: roles of $\beta$-lactamases, siderophore receptors, and penicillin binding protein 3. Antimicrob Agents Chemother. 2020;64(11):e01221-e1320.

62. Kohira N, Hackel MA, Ishioka Y, et al. Reduced susceptibility mechanism to cefiderocol, a siderophore cephalosporin, among clinical isolates from a global surveillance programme (SIDEROWT-2014). J Glob Antimicrob Resist. 2020;22:738-41. 
63. Kawai A, McElheny CL, Iovleva A, et al. Structural basis of reduced susceptibility to ceftazidime-avibactam and cefiderocol in Enterobacter cloacae due to AmpC R2 loop deletion. Antimicrob Agents Chemother. 2020;64(7):e00198-e220.

64. Sato T, Ito A, Ishioka Y, et al. Escherichia coli strains possessing a four amino acid YRIN insertion in PBP3 identified as part of the SIDERO-WT-2014 surveillance study. JAC Antimicrob Resist. 2020. https://doi.org/10.1093/jacamr/dlaa081.

65. Yamano Y, Ishibashi N, Kuroiwa M, et al. Effects of exogenous pyoverdine on the anti-pseudomonal activity of cefiderocol. In: World Microbe Forum. 2021.

66. Kawaguchi N, Katsube T, Echols R, et al. Population pharmacokinetic and pharmacokinetic/pharmacodynamic analyses of cefiderocol, a parenteral siderophore cephalosporin, in patients with pneumonia, bloodstream infection/sepsis, or complicated urinary tract infection. Antimicrob Agents Chemother. 2021. https:// doi.org/10.1128/AAC.01437-20.

67. Shionogi Inc. FETROJA (cefiderocol) for injection, for intravenous use: US prescribing information. 2020. https://www.fetroja. com/. Accessed 11 May 2021.

68. European Medicines Agency. Fetcroja $1 \mathrm{~g}$ powder for concentrate for solution for infusion: EU summary of product characteristics. 2020. https://www.ema.europa.eu/. Accessed 11 May 2021.

69. Katsube T, Wajima T, Echols R, et al. Intrapulmonary pharmacokinetics of cefiderocol in hospitalized and ventilated patients receiving standard of care antibiotics for bacterial pneumonia [abstract no. 1311]. Open Forum Infect Dis. 2020;7(Suppl 1):S668.

70. Katsube T, Saisho Y, Shimada J, et al. Intrapulmonary pharmacokinetics of cefiderocol, a novel siderophore cephalosporin, in healthy adult subjects. J Antimicrob Chemother. 2019;74(7):1971-4.

71. Kawaguchi N, Katsube T, Echols R, et al. Population pharmacokinetic analysis of cefiderocol, a parenteral siderophore cephalosporin, in healthy subjects, subjects with various degrees of renal function, and patients with complicated urinary tract infection or acute uncomplicated pyelonephritis. Antimicrob Agents Chemother. 2018;62(2):e01391-e1417.

72. Katsube T, Echols R, Arjona Ferreira JC, et al. Cefiderocol, a siderophore cephalosporin for Ggram-negative bacterial infections: pharmacokinetics and safety in subjects with renal impairment. J Clin Pharmacol. 2017;57(5):584-91.

73. Katsube T, Miyazaki S, Narukawa Y, et al. Drug-drug interaction of cefiderocol, a siderophore cephalosporin, via human drug transporters. Eur J Clin Pharmacol. 2018;74(7):931-8.

74. Portsmouth S, van Veenhuyzen D, Echols R, et al. Cefiderocol versus imipenem-cilastatin for the treatment of complicated urinary tract infections caused by Gram-negative uropathogens: a phase 2, randomised, double-blind, non-inferiority trial. Lancet Infect Dis. 2018;18(12):1319-28.

75. Wunderink RG, Matsunaga Y, Ariyasu M, et al. Cefiderocol versus high-dose, extended-infusion meropenem for the treatment of Gram-negative nosocomial pneumonia (APEKS-NP): a randomised, double-blind, phase 3, non-inferiority trial. Lancet Infect Dis. 2021;21(2):213-5.
76. Bassetti M, Echols R, Matsunaga Y, et al. Efficacy and safety of cefiderocol or best available therapy for the treatment of serious infections caused by carbapenem-resistant Gram-negative bacteria (CREDIBLE-CR): a randomised, open-label, multicentre, pathogen-focused, descriptive, phase 3 trial. Lancet Infect Dis. 2020;21(2):226-40.

77. Matsunaga Y, Sonoyama T, Casanova L, et al. Safety profile of the novel siderophore cephalosporin cefiderocol in randomized phase 2 and phase 3 clinical studies of serious Gram-negative infections [abstract no. 1292]. Open Forum Infect Dis. 2020;7(Suppl 1):S661-2.

78. European Association of Urology. EAU guidelines on urological infections. 2021. https://uroweb.org/. Accessed 11 May 2021.

79. Reason T, Gill K, Longshaw C, et al. Treatments for complicated urinary tract infections (cUTI) caused by multidrug resistant (MDR) Gram-negative (GN) pathogens: a systematic review and network meta-analysis (NMA) [abstract no. 1578]. Open Forum Infect Dis. 2020;7(Suppl 1):S787.

80. Tamma PD, Aitken SL, Bonomo RA, et al. Infectious Diseases Society of America guidance on the treatment of antimicrobial resistant gram-negative infections. 2020. https://www.idsociety. org/. Accessed 11 May 2021.

81. Torres A, Niederman MS, Chastre J, et al. International ERS/ ESICM/ESCMID/ALAT guidelines for the management of hospital-acquired pneumonia and ventilator-associated pneumonia. Eur Respir J. 2017. https://doi.org/10.1183/13993003.00582-2017.

82. Kalil AC, Metersky ML, Klompas M, et al. Management of adults with hospital-acquired and ventilator-associated pneumonia: 2016 clinical practice guidelines by the Infectious Diseases Society of America and the American Thoracic Society. Clin Infect Dis. 2016;63(5):e61-111.

83. National Institute for Health and Care Excellence. Antimicrobial prescribing: cefiderocol. 2020. https://www.nice.org.uk/. Accessed 11 May 2021.

84. European Medicines Agency. Recarbrio $500 \mathrm{mg} / 500 \mathrm{mg} / 250 \mathrm{mg}$ powder for solution for infusion: EU summary of product characteristics. 2020. https://www.ema.europa.eu/. Accessed 11 May 2021.

85. Bavaro DF, Belati A, Stufano M, et al. Cefiderocol-based combination therapy as a "rescue" treatment for severe XDR Gram-negative infections in immunocompromised or critically ill patients: a retrospective case series [abstract no. 2517]. In: 31st European Congress of Clinical Microbiology \& Infectious Diseases. 2021.

86. Tiseo G, Falcone M, Della Sala L, et al. Cefiderocol as rescue therapy for carbapenem-resistant Gram-negative infections in ICU patients: a single-center experience, Pisa, Italy 2020 [abstract no. 4507]. In: 31st European Congress of Clinical Microbiology \& Infectious Diseases. 2021.

87. Viale P, Tascini C, Dinh A, et al. Systematic review of published cases of cefiderocol use in real-world clinical practice [abstract no. 3585]. In: 31st European Congress of Clinical Microbiology \& Infectious Diseases. 2021. 